\title{
Using LTAG Based Features in Parse Reranking*
}

\author{
Libin Shen \\ Anoop Sarkar \\ Aravind K. Joshi \\ Dept. of Computer \& Info. Sci. School of Computing Science Dept. of Computer \& Info. Sci. \\ University of Pennsylvania \\ libinecis. upenn. edu \\ Simon Fraser University \\ University of Pennsylvania \\ anoopecs.sfu.ca \\ joshi@cis.upenn.edu
}

\begin{abstract}
We propose the use of Lexicalized Tree Adjoining Grammar (LTAG) as a source of features that are useful for reranking the output of a statistical parser. In this paper, we extend the notion of a tree kernel over arbitrary sub-trees of the parse to the derivation trees and derived trees provided by the LTAG formalism, and in addition, we extend the original definition of the tree kernel, making it more lexicalized and more compact. We use LTAG based features for the parse reranking task and obtain labeled recall and precision of $89.7 \% / 90.0 \%$ on WSJ section 23 of Penn Treebank for sentences of length $\leq 100$ words. Our results show that the use of LTAG based tree kernel gives rise to a $17 \%$ relative difference in $f$-score improvement over the use of a linear kernel without LTAG based features.
\end{abstract}

\section{Introduction}

Recent work in statistical parsing has explored alternatives to the use of (smoothed) maximum likelihood estimation for parameters of the model. These alternatives are distribution-free (Collins, 2001), providing a discriminative method for resolving parse ambiguity. Discriminative methods provide a ranking between multiple choices for the most plausible parse tree for a sentence, without assuming that a particular distribution or stochastic process generated the alternative parses.

\footnotetext{
"We would like to thank Michael Collins for providing the original $n$-best parsed data on which we ran our experiments and the anonymous reviewers for their comments. The second author is partially supported by NSERC, Canada (RGPIN: 264905).
}

Discriminative methods permit the use of feature functions that can be used to condition on arbitrary aspects of the input. This flexibility makes it possible to incorporate features of various of kinds. Features can be defined on characters, words, part of speech (POS) tags and context-free grammar (CFG) rules, depending on the application to which the model is applied.

Features defined on $n$-grams from the input are the most commonly used for NLP applications. Such $n$-grams can either be defined explicitly using some linguistic insight into the problem, or the model can be used to search the entire space of $n$ gram features using a kernel representation. One example is the use of a polynomial kernel over sequences. However, to use all possible $n$-gram features typically introduces too many noisy features, which can result in lower accuracy. One way to solve this problem is to use a kernel function that is tailored for particular NLP applications, such as the tree kernel (Collins and Duffy, 2001) for statistical parsing.

In addition to $n$-gram features, more complex high-level features are often exploited to obtain higher accuracy, especially when discriminative models are used for statistical parsing. For example, all possible sub-trees can be used as features (Collins and Duffy, 2002; Bod, 2003). However, most of the sub-trees are linguistically meaningless, and are a source of noisy features thus limiting efficiency and accuracy. An alternative to the use of arbitrary sets of sub-trees is to use the set of elementary trees as defined in Lexicalized Tree Adjoining Grammar (LTAG) (Joshi and Schabes, 1997). LTAG based features not only allow a more limited and a linguistically more valid set of features over sub-trees, they also provide the use of features that use discontinuous sub-trees which are outside the 
scope of previous tree kernel definitions using arbitrary sub-trees. In this paper, we use the LTAG based features in the parse reranking problem (Collins, 2000; Collins and Duffy, 2002). We use the Support Vector Machine (SVM) (Vapnik, 1999) based algorithm proposed in (Shen and Joshi, 2003) as the reranker in this paper. We apply the tree kernel to derivation trees of LTAG, and extract features from derivation trees. Both the tree kernel and the linear kernel on the richer feature set are used. Our experiments show that the use of tree kernel on derivation trees makes the notion of a tree kernel more powerful and more applicable.

\section{Lexicalized Tree Adjoining Grammar}

In this section, we give a brief introduction to the Lexicalized Tree Adjoining Grammar (more details can be found in (Joshi and Schabes, 1997)). In LTAG, each word is associated with a set of elementary trees. Each elementary tree represents a possible tree structure for the word. There are two kinds of elementary trees, initial trees and auxiliary trees. Elementary trees can be combined through two operations, substitution and adjunction. Substitution is used to attach an initial tree, and adjunction is used to attach an auxiliary tree. In addition to adjunction, we also use sister adjunction as defined in the LTAG statistical parser described in (Chiang, 2000). ${ }^{1}$ The tree resulting from the combination of elementary trees is is called a derived tree. The tree that records the history of how a derived tree is built from the elementary trees is called a derivation tree. ${ }^{2}$

We illustrate the LTAG formalism using an example.

Example 1: Pierre Vinken will join the board as a non-executive director.

The derived tree for Example 1 is shown in Fig. 1 (we omit the POS tags associated with each word to save space), and Fig. 2 shows the elementary trees for each word in the sentence. Fig. 3 is the derivation tree (the history of tree combinations). One of

\footnotetext{
${ }^{1}$ Adjunction is used in the case where both the root node and the foot node appear in the Treebank tree. Sister adjunction is used in generating modifier sub-trees as sisters to the head, e.g in basal NPs.

${ }^{2}$ Each node $\eta\langle n\rangle$ in the derivation tree is an elementary tree name $\eta$ along with the location $n$ in the parent elementary tree where $\eta$ is inserted. The location $n$ is the Gorn tree address (see Fig. 4).
}

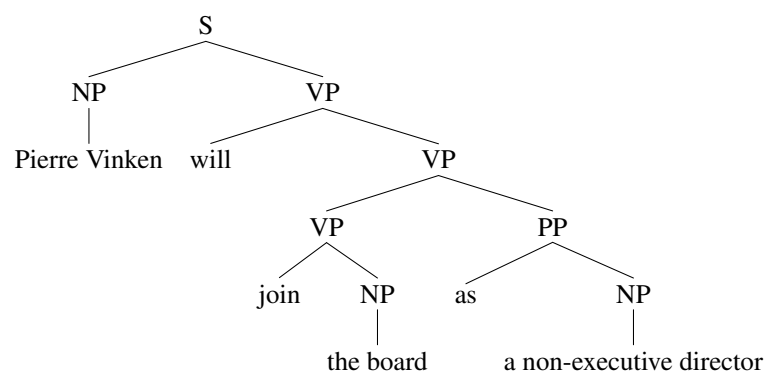

Figure 1: Derived tree (parse tree) for Example 1.
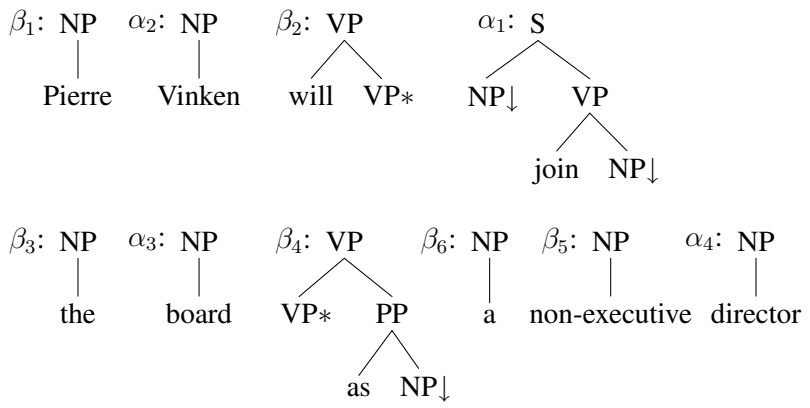

Figure 2: Elementary trees for Example 1.

the properties of LTAG is that it factors recursion in clause structure from the statement of linguistic constraints, thus making these constraints strictly local. For example, in the derivation tree of Examples 1, $\alpha_{1}($ join $)$ and $\alpha_{2}$ (Vinken) are directly connected whether there is an auxiliary tree $\beta_{2}$ (will) or not. We will show how this property affects our redefined tree kernel later in this paper. In our experiments in this paper, we only use LTAG grammars where each elementary tree is lexicalized by exactly one word (terminal symbol) on the frontier.

\section{Parse Reranking}

In recent years, reranking techniques have been successfully used in statistical parsers to rerank the output of history-based models (Black et al., 1993). In this paper, we will use the LTAG based features to improve the performance of reranking. Our motivations for using LTAG based features for reranking are the following:

- Unlike the generative model, it is trivial to incorporate features of various kinds in a reranking setting. Furthermore the nature of reranking makes it possible to use global features, 


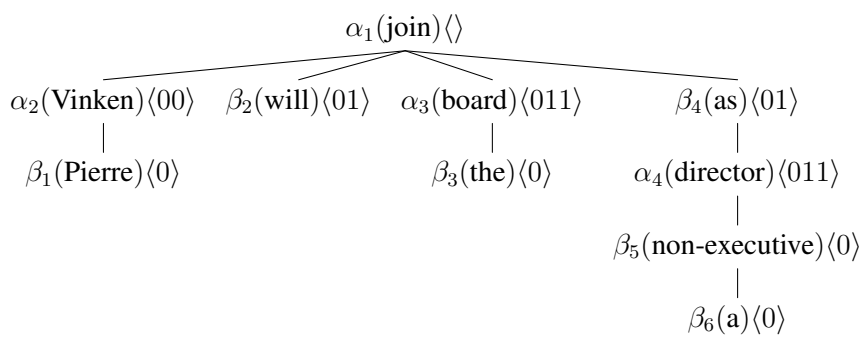

Figure 3: Derivation tree: shows how the elementary trees shown in Fig. 2 can be combined to provide an analysis for the sentence in Example 1.

which allow us to combine features that are defined on arbitrary sub-trees in the parse tree and features defined on a derivation tree.

- Several hand-crafted and arbitrary features have been exploited in the statistical parsing task, especially when parsing the WSJ Penn Treebank dataset where performance has been finely tuned over the years. Showing a positive contribution in this task will be a convincing test for the use of LTAG based features.

- The parse reranking dataset is well established. We use the dataset defined in (Collins, 2000).

In (Collins, 2000), two reranking algorithms were proposed. One was based on Markov Random Fields, and the other was based on the Boosting algorithm. In both these models, the loss functions were computed directly on the feature space. Furthermore, a rich feature set was introduced that was specifically selected by hand to target the limitations of generative models in statistical parsing.

In (Collins and Duffy, 2002), the Voted Perceptron algorithm was used for parse reranking. The

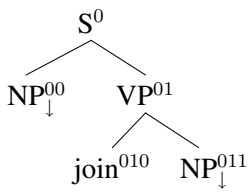

Figure 4: Example of how each node in an elementary tree has a unique node address using the Gorn notation. 0 is the root with daughters 00,01 , and so on recursively, e.g. first daughter 01 is 010 .

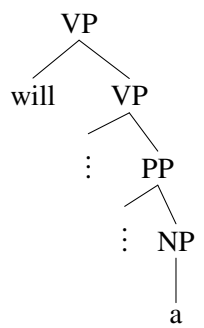

Figure 5: A sub-tree which is linguistically meaningless.

tree kernel was used to compute the number of common sub-trees of two parse trees. The features used by this tree kernel contains all the hand selected features of (Collins, 2000). It is worth mentioning that the $f$-scores reported in (Collins and Duffy, 2002) are about $1 \%$ less than the results in (Collins, 2000).

In (Shen and Joshi, 2003), a SVM based reranking algorithm was proposed. In that paper, the notion of preference kernels was introduced to solve the reranking problem. Two distinct kernels, the tree kernel and the linear kernel were used with preference kernels.

\section{Using LTAG Based Features}

\subsection{Motivation}

While the tree kernel is an easy way to compute similarity between two parse trees, it takes too many linguistically meaningless sub-trees into consideration.

Let us consider the example sentence in Example 1. The parse tree, or derived tree, for this sentence is shown in Fig. 1. Fig. 5 shows one of the linguistically meaningless sub-trees. The number of meaningless sub-trees is a misleading measure for discriminating good parse trees from bad. Furthermore, the number of meaningless sub-trees is far greater than the number of useful sub-trees. This limits both efficiency and accuracy on the test data. The use of unwanted sub-trees greatly increases the hypothesis space of a learning machine, and thus decreases the expected accuracy on test data. In this work, we consider the hypothesis that linguistically meaningful sub-trees reveal correlations of interest and therefore are useful in stochastic models.

We notice that each sub-tree of a derivation tree is linguistically meaningful because it represents a valid sub-derivation. We claim that derivation trees 
provide a more accurate measure of similarity between two parses. This is one of the motivations for applying tree kernels to derivation trees. Note that the use of features on derivation trees is different from the use of features on dependency graphs, derivation trees include many complex patterns of tree names and attachment sites and can represent word to word dependencies that are not possible in traditional dependency graphs.

For example, the derivation tree for Example 1 with and without optional modifiers such as $\beta_{4}(a s)$ are minimally different. In contrast, in derived (parse) trees, there is an extra VP node which changes quite drastically the set of sub-trees with and without the PP modifier. In addition, using only sub-trees from the derived tree, we cannot represent a common sub-tree that contains only the words Vinken and join since this would lead to a discontinuous sub-tree. However, LTAG based features can represent such cases trivially.

The comparison between (Collins, 2000) and (Collins and Duffy, 2002) in $\S 3$ shows that it is hard to add new features to improve performance. Our hypothesis is that the LTAG based features provide a novel set of abstract features that complement the hand selected features from (Collins, 2000) and the LTAG based features will help improve performance in parse reranking.

\subsection{Extracting Derivation Trees}

Before we can use LTAG based features we need to obtain an LTAG derivation tree for each parse tree under consideration by the reranker. Our solution is to extract elementary trees and the derivation tree simultaneously from the parse trees produced by an $n$-best statistical parser. Our training and test data consists of $n$-best output from the Collins parser (see (Collins, 2000) for details on the dataset). Since the Collins parser uses a lexicalized contextfree grammar as a basis for its statistical model, we obtain parse trees that are of the type shown in Fig. 6. From this tree we extract elementary trees and derivation trees by recursively traversing the spine of the parse tree. The spine is the path from a nonterminal lexicalized by a word to the terminal symbol on the frontier equal to that word. Every sub-tree rooted at a non-terminal lexicalized by a different word is excised from the parse tree and recorded into

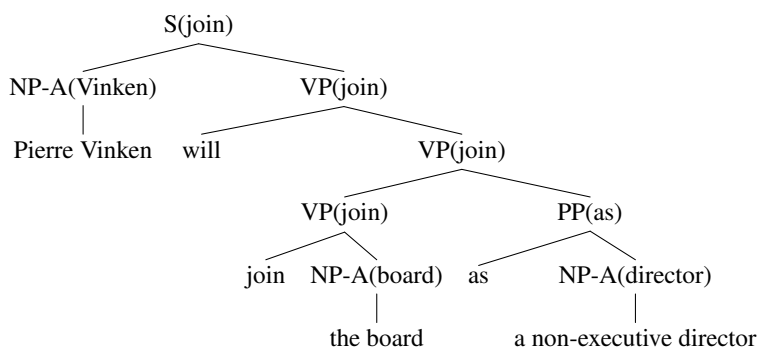

Figure 6: Sample output parse from the Collins parser. Each non-terminal is lexicalized by the parsing model. $-A$ marks arguments recovered by the parser.

the derivation tree as a substitution. Repeated nonterminals on the spine (e.g. VP(join) ... VP(join) in Fig. 6) are excised along with the sub-trees hanging off of it and recorded into the derivation tree as an adjunction. The only other case is those subtrees rooted at non-terminals that are attached to the spine. These sub-trees are excised and recorded into the derivation tree as cases of sister adjunction. Each sub-tree excised is recursively analyzed with this method, split up into elementary trees and then recorded into the derivation tree. The output of our algorithm for the input parse tree in Fig. 6 is shown in Fig. 2 and Fig. 3. Our algorithm is similar to the derivation tree extraction explained in (Chiang, 2000), except we extract our LTAG from $n$-best sets of parse trees, while in (Chiang, 2000) the LTAG is extracted from the Penn Treebank. ${ }^{3}$ For other techniques for LTAG grammar extraction see (Xia, 2001; Chen and Vijay-Shanker, 2000).

\subsection{Using Derivation Trees}

In this paper, we have described two models to employ derivation trees. Model 1 uses tree kernels on derivation trees. In order to make the tree kernel more lexicalized, we extend the original definition of the tree kernel, which we will describe below. Model 2 abstracts features from derivation trees and uses them with a linear kernel.

In Model 1, we combine the SVM results of the tree kernel on derivation trees with the SVM results given by a linear kernel based on features on the derived trees.

\footnotetext{
${ }^{3}$ Also note that the path from the root node to the foot node in auxiliary trees can be greater than one (for trees with $S$ roots).
} 
In Model 2, the vector space of the linear kernel consists of both LTAG based features defined on the derived trees and features defined on the derivation tree. The following LTAG features have been used in Model 2.

- Elementary tree. Each node in the derivation tree is used as a feature.

- Bigram of parent and its child. Each pair of parent elementary tree and child elementary tree, as well as the type of operation (substitution, adjunction or sister adjunction) and the Gorn address on parent (see Fig. 4) is used as a feature.

- Lexicalized elementary tree. Each elementary tree associated with its lexical item is used as a feature.

\section{- Lexicalized bigram. In Bigram of parent and} its child, each elementary tree is lexicalized (we use closed class words, e.g. adj, adv, prep, etc. but not noun or verb).

\subsection{Lexicalized Tree Kernel}

In (Collins and Duffy, 2001), the notion of a tree kernel is introduced to compute the number of common sub-trees of two parse trees. For two parse trees, $p_{1}$ and $p_{2}$, the tree kernel $\operatorname{Tree}\left(p_{1}, p_{2}\right)$ is defined as:

$$
\operatorname{Tree}\left(p_{1}, p_{2}\right)=\sum_{\substack{n_{1} \text { in } p_{1} \\ n_{2} \text { in } p_{2}}} T\left(n_{1}, n_{2}\right)
$$

The recursive function $T$ is defined as follows: If $n_{1}$ and $n_{2}$ have the same bracketing tag (e.g. $S, N P, V P$, ...) and the same number of children,

$$
T\left(n_{1}, n_{2}\right)=\lambda \prod_{i}\left(1+T\left(n_{1 i}, n_{2 i}\right)\right),
$$

where, $n_{k i}$ is the $i$ th child of the node $n_{k}, \lambda$ is a weight coefficient used to control the importance of large sub-trees and $0<\lambda \leq 1$.

If $n_{1}$ and $n_{2}$ have the same bracketing tag but different number of children, $T\left(n_{1}, n_{2}\right)=\lambda$. If they don't have the same bracketing tag, $T\left(n_{1}, n_{2}\right)=0$.

In (Collins and Duffy, 2002), lexical items are all located at the leaf nodes of parse trees. Therefore
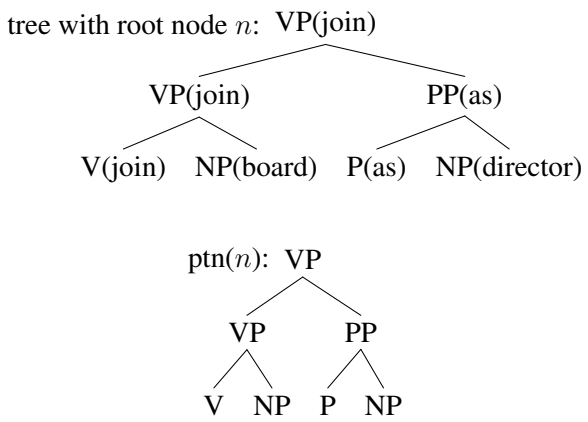

$\operatorname{lex}(n)$ : (join, join, as)

Figure 7: A lexicalized sub-tree rooted at $n$ and its decomposition into a pattern, $\operatorname{ptn}(n)$ and corresponding vector of lexical information, $\operatorname{lex}(n)$.

sub-trees that do not contain any leaf node are not lexicalized. Furthermore, due to the introduction of parameter $\lambda$, lexical information is almost ignored for sub-trees whose root node is not close to the leaf nodes, i.e. sub-trees rooted at $S$ node.

In order to make the tree kernel more lexicalized, we associate each node with a lexical item. For example, Fig. 7 shows a lexicalized sub-tree and its decomposition into features. As shown in Fig. 7 the lexical information lex(t) extracted from the lexicalized tree consists of words from the root and its immediate children. This is because we wish to ignore irrelevant lexicalizations such as $N P($ board $)$ in Fig. 7.

A lexicalized sub-tree rooted on node $n$ is split into two parts. One is the pattern tree of $n, p t n(n)$. The other is the vector of lexical information of $n$, lex $(n)$, which contains the lexical items of the root node and the children of the root.

For two tree nodes $n_{1}$ and $n_{2}$, the recursive function $\operatorname{LT}\left(n_{1}, n_{2}\right)$ used to compute the lexicalized tree kernel is defined as follows.

$$
\begin{aligned}
L T\left(n_{1}, n_{2}\right) & =\left(1+C n t\left(\operatorname{lex}\left(n_{1}\right), \operatorname{lex}\left(n_{2}\right)\right)\right) \\
& \times T^{\prime}\left(p \operatorname{tn}\left(n_{1}\right), p \operatorname{tn}\left(n_{2}\right)\right)
\end{aligned}
$$

where $T^{\prime}$ is the same as the original recursive function $T$ defined in (2), except that $T$ is defined on parse tree nodes, while $T^{\prime}$ is defined on patterns of parse tree nodes. $\operatorname{Cnt}(x, y)$ counts the number of common elements in vector $x$ and $y$. For example, Cnt $(($ join, join, as $),($ join, join, in $))=2$, since 
2 elements of the two vectors are the same.

It can be shown that the lexicalized tree kernel counts the number of common sub-trees that meet the following constraints.

- None or one node in the sub-tree is lexicalized

- The lexicalized node is the root node or a child of the root, if applicable.

Therefore our new tree kernel is more lexicalized. On the other hand, it immediately follows that the lexicalized tree kernel is well-defined. It means that we can embed the lexicalized tree kernel into a high dimensional space. The proof is similar to the proof for the tree kernel in (Collins and Duffy, 2001).

Another important advantage of the lexicalized tree kernel is that it is more compressible. It is noted in (Collins and Duffy, 2001) that training trees can be combined by sharing sub-trees to speed up the test. As far as the lexicalized tree kernel is concerned, the pattern trees are more compressible because there is no lexical item at the leaf nodes of pattern trees. Lexical information can be attached to the nodes of the result pattern forest. In our experiment, we select five parses from each sentence in Collins' training data and represent these parses with shared structure. The number of the nodes in the pattern forest is only $1 / 7$ of the total number of the nodes the selected parse trees.

\subsection{Tree Kernel for Derivation Trees}

In order to apply the (lexicalized) tree kernel to derivation trees, we need to make some modifications to the original recursive definition of the tree kernel.

For derivation trees, the recursive function is triggered if the two root nodes have the same nonlexicalized elementary tree (sometimes called supertag). Note that these two nodes will have the same number of children which are initial trees (auxiliary trees are not counted). In comparison, the recursive function in (2), $T\left(n_{1}, n_{2}\right)$ is computed if and only if $n_{1}$ and $n_{2}$ have the same bracketing tag and they have the same number of children.

For each node, its children are attached with one of the two distinct operations, substitution or adjunction. For substituted children, the computation of the tree kernel is almost the same as that for CFG parse tree. However, there is a problem with the adjoined children. Let us first have a look at a sentence in Penn Treebank.

Example 2: COMMERCIAL PAPER placed directly by General Motors Acceptance Corp.: $8.55 \%$ 30 to 44 days; $8.25 \% 45$ to 59 days; $8.45 \% 60$ to 89 days; $8 \% 90$ to 119 days; $7.90 \% 120$ to 149 days; $7.80 \% 150$ to 179 days; $7.55 \% 180$ to 270 days.

In this example, seven sub-trees of the same type are sister adjoined to the same place of an initial tree. So the number of common sub-trees increases dramatically if the tree kernel is applied on two similar parses of this sentence. Experimental evidence indicates that this is harmful to accuracy. Therefore, for derivation trees, we are only interested in sub-trees that contain at most 2 adjunction branches for each node. The number of constrained common sub-trees for the derivation tree kernel can be computed by the recursive function $D T$ over derivation tree nodes $n_{1}, n_{2}$ :

$$
\begin{aligned}
D T\left(n_{1}, n_{2}\right) & =\left(1+A_{1}\left(n_{1}, n_{2}\right)+A_{2}\left(n_{1}, n_{2}\right)\right) \\
& \times T^{\prime \prime}\left(\operatorname{sub}\left(n_{1}\right), \operatorname{sub}\left(n_{2}\right)\right)
\end{aligned}
$$

where $\operatorname{sub}\left(n_{k}\right)$ is the sub-tree of $n_{k}$ in which children adjoined to the root of $n_{k}$ are pruned. T" is similar to the original recursive function $T$ defined in (2), but it is defined on derivation tree nodes recursively. $A_{1}$ and $A_{2}$ are used to count the number of common sub-trees whose root nodes only contain one or two adjunction children respectively.

$$
A_{1}\left(n_{1}, n_{2}\right)=\sum_{i, j} D T\left(a_{1 i}, a_{2 j}\right),
$$

where, $a_{1 i}$ is the $i$ th adjunct of $n_{1}$, and $a_{2 j}$ is the $j$ th adjunct of $n_{2}$. Similarly, we have:

$$
A_{2}\left(n_{1}, n_{2}\right)=\sum_{i<k, j<l} D T\left(a_{1 i}, a_{2 j}\right) \cdot \operatorname{DT}\left(a_{1 k}, a_{2 l}\right)
$$

The tree kernel for derivation trees is a well-defined kernel function because we can easily define an embedding space according to the definition of the new tree kernel. By substituting $D T$ for $T^{\prime}$ in (3), we obtain the lexicalized tree kernel for LTAG derivation trees (using $L T$ in (1)).

\section{Experiments}

As described above, we use the SVM based voting algorithm (Shen and Joshi, 2003) in our reranking 
experiments. We use preference kernels and pairwise parse trees in our reranking models.

We use the same data set as described in (Collins, 2000). Section 2-21 of the Penn WSJ Treebank are used as training data, and section 23 is used for final test. The training data contains around 40,000 sentences, each of which has 27 distinct parses on average. Of the 40,000 training sentences, the first 36,000 are used to train SVMs. The remaining 4,000 sentences are used as development data.

Due to the computational complexity of SVM, we have to divide training data into slices to speed up training. Each slice contain two pairs of parses from every sentence. Specifically, slice $i$ contains positive samples $\left(\left(\tilde{p}_{k}, p_{k i}\right),+1\right)$ and negative samples $\left(\left(p_{k i}, \tilde{p}_{k}\right),-1\right)$, where $\tilde{p}_{k}$ is the best parse for sentence $k, p_{k i}$ is the parse with the $i$ th highest loglikelihood in all the parses for sentence $k$ and it is not the best parse (Shen and Joshi, 2003). There are about 60000 samples in each slice in average.

For the tree kernel SVMs of Model 1, we take 3 slices as a chunk, and train an SVM for each chunk. Due to the limitation of computing resource, we have only trained on 3 chunks. The results of tree kernel SVMs are combined with simple combination. Then the outcome is combined with the result of the linear kernel SVMs trained on features extracted from the derived trees which are reported in (Shen and Joshi, 2003). For each parse, the number of the brackets in it and the log-likelihood given by Collins' parser Model 2 are also used in the computation of the score of a parse. For each parse $p$, its score $S c o(p)$ is defined as follows:

$S c o(p)=M_{T}(p)+\gamma \cdot M_{L}(p)+\beta \cdot l(p)+\alpha \cdot b(p)$

where $M_{T}(p)$ is the output of the tree kernel SVMs, $M_{L}(p)$ is the output of linear kernel SVMs, $l(p)$ is the log-likelihood of parse $p$, and $b(p)$ is the number of brackets in parse $p$. We noticed that the SVM systems prefers to give higher scores to the parses with less brackets. As a result, the system has a high precision but a low recall. Therefore, we take the number of brackets, $b(p)$, as a feature to make the recall and precision balanced. The three weight parameters are tuned on the development data.

The results are shown in Table 1. With Model 1, we achieve LR/LP of $89.7 \% / 90.0 \%$ on sentences

\begin{tabular}{|c|c|c|c|c|c|}
\hline \multicolumn{7}{|c|}{$\leq 40$ Words (2245 sentences) } \\
\hline Model & LR & LP & CBs & 0 CBs & 2 CBs \\
\hline CO99 & $88.5 \%$ & $88.7 \%$ & 0.92 & $66.7 \%$ & $87.1 \%$ \\
\hline CO00 & $90.1 \%$ & $90.4 \%$ & 0.73 & $70.7 \%$ & $89.6 \%$ \\
\hline CD02 & $89.1 \%$ & $89.4 \%$ & 0.85 & $69.3 \%$ & $88.2 \%$ \\
\hline SJ 03 & $89.9 \%$ & $90.3 \%$ & 0.75 & $71.7 \%$ & $89.4 \%$ \\
\hline M1 & $90.2 \%$ & $90.5 \%$ & 0.72 & $72.3 \%$ & $90.0 \%$ \\
\hline M2 & $89.8 \%$ & $90.3 \%$ & 0.76 & $71.6 \%$ & $89.6 \%$ \\
\hline \hline \multicolumn{7}{|c|}{$\leq 100$ Words (2416 sentences) } \\
\hline Model & LR & LP & CBs & 0 CBs & 2 CBs \\
\hline CO99 & $88.1 \%$ & $88.3 \%$ & 1.06 & $64.0 \%$ & $85.1 \%$ \\
\hline CO00 & $89.6 \%$ & $89.9 \%$ & 0.87 & $68.3 \%$ & $87.7 \%$ \\
\hline CD02 & $88.6 \%$ & $88.9 \%$ & 0.99 & $66.5 \%$ & $86.3 \%$ \\
\hline SJ 03 & $89.4 \%$ & $89.8 \%$ & 0.89 & $69.2 \%$ & $87.6 \%$ \\
\hline M1 & $89.7 \%$ & $90.0 \%$ & 0.86 & $70.0 \%$ & $88.2 \%$ \\
\hline M2 & $89.3 \%$ & $89.8 \%$ & 0.89 & $69.1 \%$ & $87.7 \%$ \\
\hline
\end{tabular}

Table 1: Results on section 23 of the WSJ Treebank. $\mathrm{LR} / \mathrm{LP}=$ labeled recall/precision. $\mathrm{CBs}=\mathrm{av}$ erage number of crossing brackets per sentence. 0 CBs, 2 CBs are the percentage of sentences with 0 or $\leq 2$ crossing brackets respectively. CO99 = (Collins, 1999) Model 2. CO00 $=($ Collins, 2000). CD02 $=($ Collins and Duffy, 2002). SJ03 $=$ linear kernel of (Shen and Joshi, 2003). M1=Model 1. M2=Model 2.

with $\leq 100$ words. Our results show a $17 \%$ relative difference in $f$-score improvement over the use of a linear kernel without LTAG based features (Shen and Joshi, 2003). In addition, we also get non-trivial improvement on the number of crossing brackets. These results verify the benefit of using LTAG based features and confirm the hypothesis that LTAG based features provide a novel set of abstract features that complement the hand selected features from (Collins, 2000). Our results on Model 1 show a $1 \%$ error reduction on the previous best reranking result using the dataset reported in (Collins, 2000). Also, Model 1 provides a $10 \%$ reduction in error over (Collins and Duffy, 2002) where the features from tree kernel were over arbitrary sub-trees.

For Model 2, we first train 22 SVMs on 22 distinct slices. Then we combine the results of individual SVMs with simple combination. However, the overall performance does not improve. But we notice that the use of LTAG based features gives rise to 


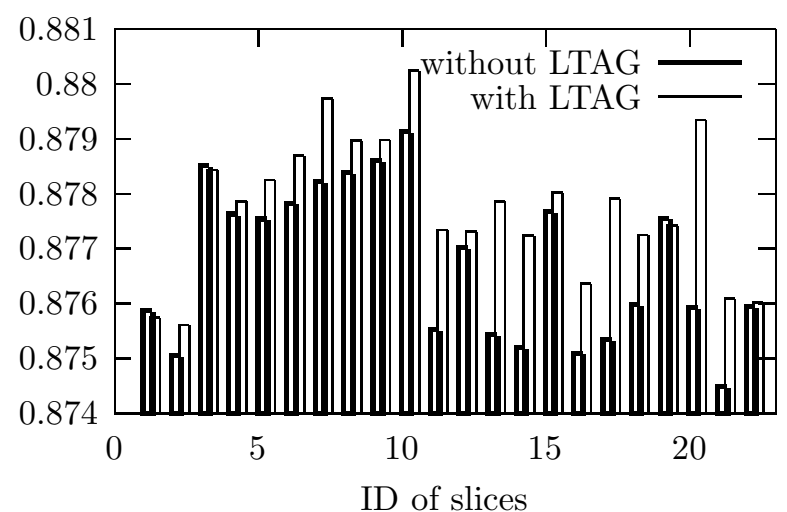

Figure 8: Comparison of performance of individual SVMs in Model 2: with and without LTAG based features. $\mathrm{X}$-axis stands for the ID of the slices on which the SVMs are trained.Y-axis stands for the $f$ score.

improvement on most of the single SVMs, as shown in Fig. 8.

We think there are several reasons to account for why our Model 2 doesn't work as well for the full task when compared with Model 1. Firstly, the training slice is not large enough. Local optimization on each slice does not result in global optimization (as seen in Fig. 8). Secondly, the LTAG based features that we have used in the linear kernel in Model 2 are not as useful as the tree kernel in Model $1 .^{4}$ The last reason is that we do not set the importance of LTAG based features. One shortcoming of kernel methods is that the coefficient of each feature must be set before the training (Herbrich, 2002). In our case, we do not tune the coefficients for the LTAG based features in Model 2.

\section{Conclusions and Future Work}

In this paper, we have proposed methods for using LTAG based features in the parse reranking task. The experimental results show that the use of LTAG based features gives rise to improvement over already finely tuned results. We used LTAG based features for the parse reranking task and obtain labeled recall and precision of $89.7 \% / 90.0 \%$ on WSJ section 23 of Penn Treebank for sentences of length $\leq$ 100 words. Our results show that the use of LTAG

\footnotetext{
${ }^{4}$ In Model 1, we implicitly take every sub-tree of the derivation trees as a feature, but in Model 2, we only consider a small set of sub-trees in a linear kernel.
}

based tree kernel gives rise to a $17 \%$ relative difference in $f$-score improvement over the use of a linear kernel without LTAG based features. In future work, we will use some light-weight machine learning algorithms for which training is faster, such as variants of the Perceptron algorithm. This will allow us to use larger training data chunks and take advantage of global optimization in the search for relevant features.

\section{References}

E. Black, F. Jelinek, J. Lafferty, Magerman D. M., R. Mercer, and S. Roukos. 1993. Towards history-based grammars: Using richer models for probabilistic parsing. In Proc. of the ACL 1993.

R. Bod. 2003. An Efficient Implementation of a New DOP Model. In Proc. of EACL 2003, Budapest.

J. Chen and K. Vijay-Shanker. 2000. Automated Extraction of TAGs from the Penn Treebank. In Proc. of the 6th IWPT.

D. Chiang. 2000. Statistical Parsing with an AutomaticallyExtracted Tree Adjoining Grammar. In Proc. of ACL-2000.

M. Collins and N. Duffy. 2001. Convolution kernels for natural language. In Proc. of the 14th NIPS.

M. Collins and N. Duffy. 2002. New ranking algorithms for parsing and tagging: Kernels over discrete structures, and the voted perceptron. In Proc. of ACL 2002.

M. Collins. 1999. Head-Driven Statistical Models for Natural Language Parsing. Ph.D. thesis, University of Pennsylvania.

M. Collins. 2000. Discriminative reranking for natural language parsing. In Proc. of 7th ICML.

M. Collins. 2001. Parameter estimation for statistical parsing models: Theory and practice of distribution-free methods. In Proc. of IWPT 2001. Invited Talk at IWPT 2001.

R. Herbrich. 2002. Learning Kernel Classifiers: Theory and Algorithms. MIT Press.

A. K. Joshi and Y. Schabes. 1997. Tree-adjoining grammars In G. Rozenberg and A. Salomaa, editors, Handbook of Formal Languages, volume 3, pages 69 - 124. Springer.

L. Shen and A. K. Joshi. 2003. An SVM based voting algorithm with application to parse reranking. In Proc. of CoNLL 2003

V. N. Vapnik. 1999. The Nature of Statistical Learning Theory. Springer, 2nd edition.

F. Xia. 2001. Investigating the Relationship between Grammars and Treebanks for Natural Languages. Ph.D. thesis, University of Pennsylvania, Philadelphia, PA. 